\title{
PTPRD silencing by DNA hypermethylation decreases insulin receptor signaling and leads to type 2 diabetes
}

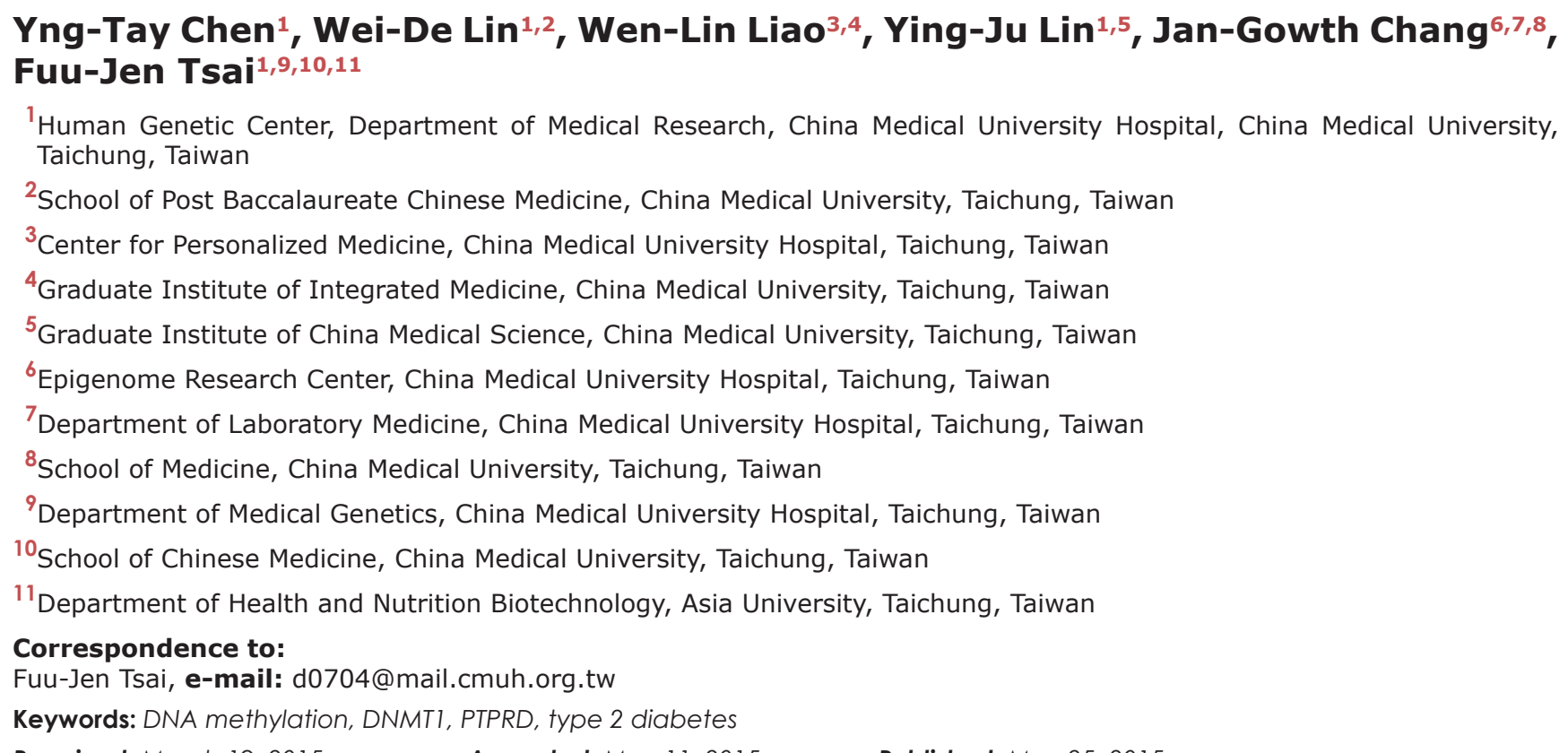

Published: May 25, 2015

\section{ABSTRACT}

Genome-wide association study (GWAS) data showed that the protein tyrosine phosphatase receptor type delta (PTPRD) is associated with increased susceptibility to type 2 diabetes (T2D) in Han Chinese. A replication study indicated that PTPRD is involved in the insulin signaling pathway; however, the underlying mechanism remains unclear. We evaluated PTPRD expression in patients with T2D and controls. PTPRD expression levels were lower in patients and were correlated with the duration of the disease. Overexpression of the human insulin receptor PPAR $\gamma 2$ in HepG 2 cells induced overexpression of PTPRD and the insulin receptor. PTPRD knockdown, using a shRNA, resulted in down-regulation of the insulin receptor. These results indicate that PTPRD activates PPAR $\gamma 2$ in the insulin signaling pathway. Similar results for PTPRD expression were found using a T2D mouse model. Silencing of PTPRD was caused by DNA methylation in T2D mice and patients, and correlated with DNMT1 expression. Furthermore, we showed that a DNMT1 SNP (rs78789647) was correlated with susceptibility to T2D. This study shows for the first time that DNMT1 caused PTPRD DNA hypermethylation and induced insulin signaling silencing in T2D patients. Our findings contribute to a better understanding of the crucial roles of these regulatory elements in human T2D.

\section{INTRODUCTION}

Type 2 diabetes (T2D) is a complex disorder that is characterized by hyperglycemia, caused by impaired pancreatic $\beta$-cell function, decreased insulin action, and increased glucose output by the liver [1-3]. Both genetic and environmental factors contribute to the pathogenesis of T2D [4]. The disease is considered a polygenic disorder in which each genetic variant confers a partial and additive effect. Only $5-10 \%$ of the T2D cases are due to single-gene defects; these include maturity-onset diabetes of the young (MODY), insulin resistance syndromes, mitochondrial diabetes, and neonatal diabetes [5-7]. Inherited variations have been identified from studies of 
monogenic diabetes and have provided insights into $\beta$-cell physiology, insulin release, and action of insulin in fat, muscle, and liver [8].

Tsai et al. [9] initially identified the rs17584499 $\mathrm{T}$ allele in the protein tyrosine phosphatase receptor type delta $(P T P R D)$ gene as the allele with the strongest association with type 2 diabetes in Han Chinese. Protein tyrosine phosphatases (PTPases) stimulate growth, proliferation, differentiation, cell cycle, and survival [10]. PTPRD is one of the most frequently inactivated genes across human cancers, including glioblastoma multiforme [11]. PTPRD was shown to be a tumor suppressor, as loss of PTPRD caused aberrant STAT3 activation in gliomas, resulting in glioma progression [12]. Epigenetic silencing of PTPRD was observed in glioblastoma [12]. The $P T P R D$ genetic variant was suggested to be associated with progression to diabetes in Han Chinese, most likely through enhanced insulin resistance [13]. Recent studies have shown that these epigenetic factors contribute to the pathogenesis of T2D. However, until now, the exact roles of epigenetic factors and PTPRD in T2D onset and development remain unclear.

To gain insight into PTPRD function and the mechanism by which it activates the insulin signaling pathway, we examined the changes in PTPRD epigenetics in $\mathrm{T} 2 \mathrm{D}$.

\section{RESULTS}

\section{Lower expression of PTPRD in patients with T2D}

GWAS data previously indicated that PTPRD is involved in T2D. However, the exact role of PTPRD in T2D is still unclear. First, we checked PTPRD mRNA levels in blood samples of patients and controls. The relative $P T P R D$ mRNA level was significantly lower in T2D patients than in controls ( 1 versus $0.07, P<0.05$, Fig. 1A). In addition, relative PTPRD mRNA expression levels were significantly lower in patients with T2D with disease duration of less than 5, 10, 15, and longer than 15 years than those of patients with disease duration less than 3 years. PTPRD mRNA levels decreased with the disease duration ( 1 versus $0.64,0.71,0.40$, and 0.37 respectively, $P<0.05$, Fig. 1B).

\section{PTPRD is involved in the insulin signaling pathway}

Little is known about the involvement of PTPRD in the insulin signaling pathway. Therefore, we used a cell model to analyze this further. We constructed a plasmid containing a fragment of human PPAR 2 (pSG5-hPPAR 2 ) for transient transfection of HepG2 cells. The results indicated that expression of human $P P A R \gamma 2$ induced the overexpression of PTPRD and the insulin receptor (Fig. 2A). We used shRNA to knock down PTPRD expression in HepG2 cells and found that the insulin receptor was simultaneously down-regulated (Fig. 2B). These results indicate that PTPRD is involved in the insulin signaling pathway.

\section{DNA hypermethylation induces PTPRD silencing in T2D mice}

We used a T2D mouse model for a time course experiment. We used 6-week-old mice as representative for the early disease stage, 16-week-old for the middle stage, and 42-week-old for the late stage. Results showed that PTPRD protein expression was significantly lower in late stage mice compared with that of early stage, middle stage, and control mice (Fig. 3A). The relative PTPRD mRNA levels decreased with the disease stage (KK versus yKK at 6,16 , and 42 weeks were $1.04,0.41$, and 0.04 respectively, $P<0.05$, Fig. 3B). These results indicated that the decrease in PTPRD expression was not caused by post-translational modification, but might be suppressed DNA transcription level. Therefore, we examined the methylation status of the PTPRD promoter, using methylation specific PCR (MSP). The result showed that the PTPRD promoter was methylated in middle and late stage T2D mice, but not in early stage T2D mice (Fig. 3C).

\section{DNMT1 induces PTPRD DNA methylation in diseased mice}

Next, we wanted to analyze which DNA methyltransferase caused PTPRD methylation in T2D mice. Therefore, we checked DNMT1, DNMT3A, $D N M T 3 B$, and DNMT3L mRNA levels in diseased mice. Results indicated that DNMT1 expression was significantly higher in later stage T2D mice (KK versus yKK at 6,16 , and 42 weeks were $0.86,0.95$, and 2.44 respectively, $P<0.05$, Fig. 3D), but that of $D N M T 3 A, D N M T 3 B$, and $D N M T 3 L$ was unaffected.

\section{PTPRD hypermethylation correlates with T2D progression}

We then investigated PTPRD methylation during disease progression, using MSP. Patients with T2D with disease duration less than 3, 5, 10, 15 years, and longer than 15 years showed hypermethylation in the PTPRD DNA promoter site (Fig. 4). Hypermethylation was observed at the onset of the disease and throughout the disease progression.

\section{DNMT1 induces PTPRD DNA methylation in patients}

In this study, the analysis of single nucleotide polymorphism (SNP) associated with DNA 
(A)

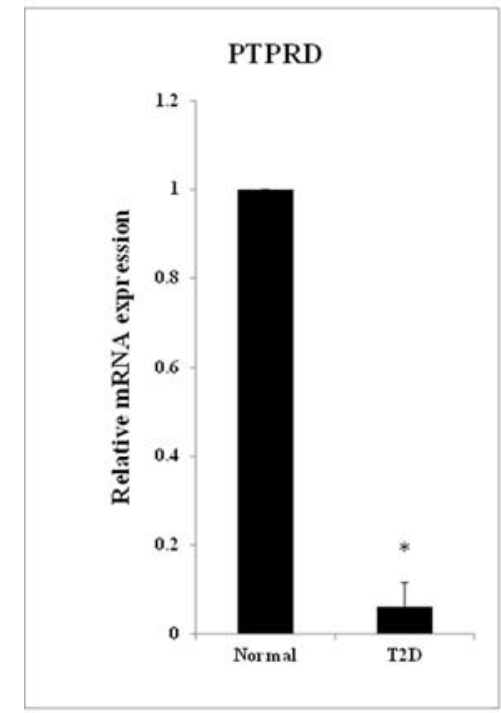

(B)

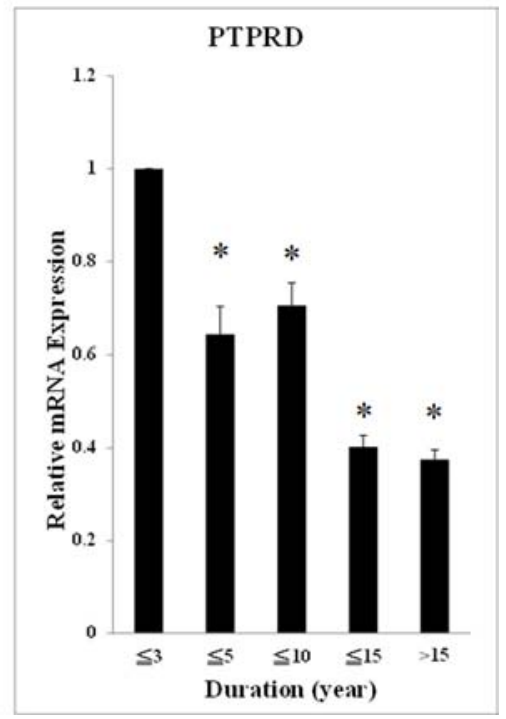

Figure 1: PTPRD mRNA was lower expression in T2D patients. A. mRNA from 94 T2D patients and 98 normal controls was used for qRT-PCR. Expression levels are expressed relative to controls. B. The 94 patients with T2D were divided into 5 groups according to the disease duration: (1) less than 3 years $(N=19)$, (2) less than 5 years $(N=16)$, (3) less than 10 years $(N=27)$, (4) less than 15 years $(N=17)$, and (5) longer than 15 years $(N=15)$. The relative mRNA expression of group (2), (3), (4), and (5) versus to group (1) were $0.64 \pm 0.06,0.71 \pm 0.05,0.40 \pm 0.03$, and $0.37 \pm 0.02$, respectively $(P<0.05)$.

(A)

\section{Vector pSG5-PPARr2}

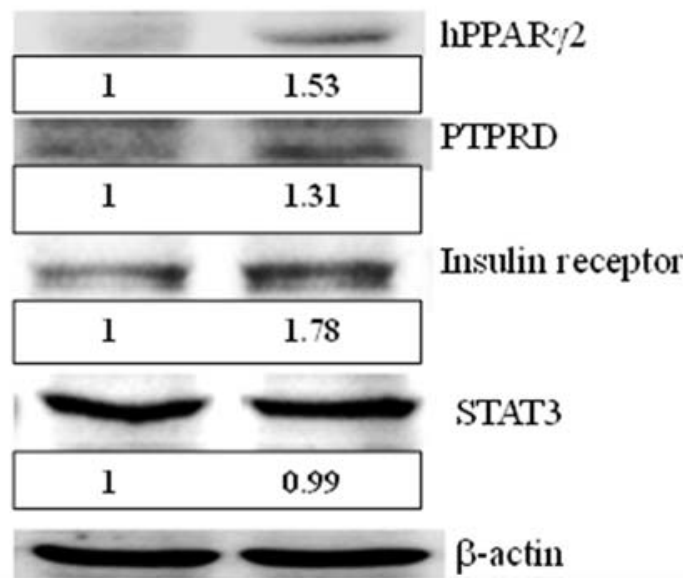

(B)

\section{Vector shRNA-PTPRD}

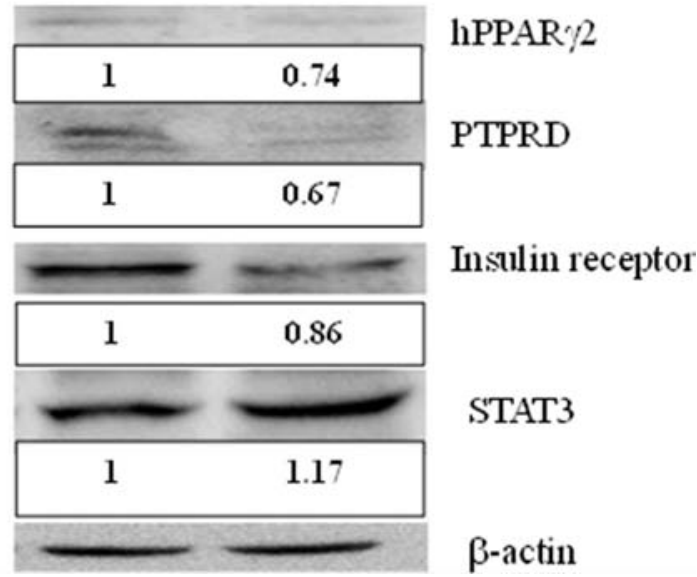

Figure 2: PTPRD is involved in the insulin signaling pathway. A. HepG2 cells were transfected with pSG5 vector and with pSG5-h-PPAR $\gamma 2$ for 48 h. PPAR 2 overexpression (1.53 fold versus vector only) induces the overexpression of PTPRD and insulin receptor 1.31 and 1.78 fold versus vector only, respectively. B. HepG2 cells were transfected with shRNA for 48 h. PTPRD knockdown (0.67 fold versus vector only) induced down-regulation of the PPAR 2 and insulin receptor ( 0.74 and 0.86 fold versus vector only, respectively). Moreover, STAT3 was induced 1.17 fold versus vector only.

methyltransferases DNMT1, DNMT3A, DNMT3B, and DNMT3L was performed on 1924 patients with T2D and 3602 controls (Table S1-S4). For SNP rs78789647, the frequency of the "TT" genotype was higher in patients $(0.52 \%)$ than in controls $(0.28 \%)$ (Table 1$)$. The frequency of the "TC" genotype was higher in patients $(14.09 \%)$ than in controls (12.08\%). In comparison with the "CC" genotype, the odds ratio (OR) of "TT" was $1.92(95 \% \mathrm{CI}=0.80-4.63, P<0.05)$. The allelic frequency of " $\mathrm{T}$ " was higher in patients $(7.56 \%)$ than in controls $(6.32 \%)$. In comparison to the " $\mathrm{C}$ " allele, the OR for the "T" allele was $1.21(95 \% \mathrm{CI}=1.04-1.41, P<0.05)$. All differences were found to be statistically significant. We found a DNMT1 SNP (rs78789647) that correlated 
(B)

(A)

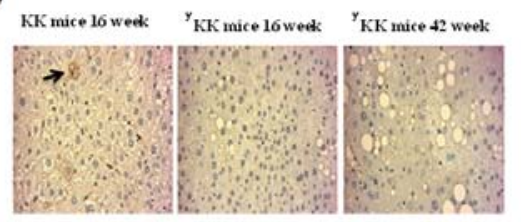

(C)

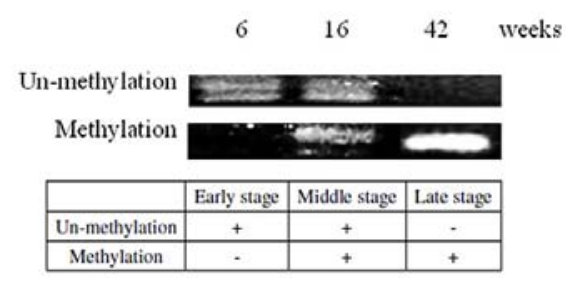

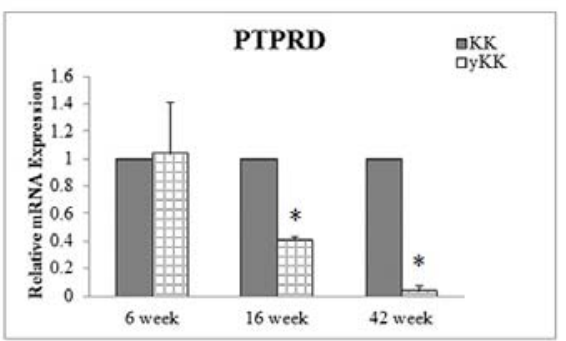

(D)

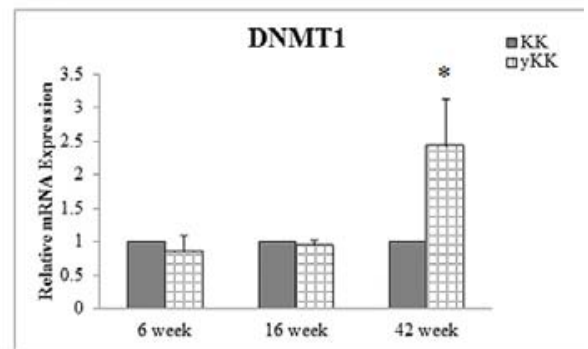

Figure 3: PTPRD protein was inhibition by T2D development duration in mice. A. Immunocytochemical staining of liver tissues shows that PTPRD protein is expressed in 16-week-old KK mice, but not in 16- and 42-week-old ${ }^{\mathrm{K}} \mathrm{KK}$ mice. B. PTPRD mRNA expression in liver tissues is not different in 6-week-old KK and yKK mice. However, expression is significantly lower in 16-and 42-week-old ${ }^{\mathrm{y}} \mathrm{KK}$ mice than in KK mice. C. PTPRD DNA showed un-methylation in 6- and 16-week-old ${ }^{y} \mathrm{KK}$ mice, but not in 42 -week-old ${ }^{\mathrm{y}} \mathrm{KK}$ mice. $P T P R D$ is methylated in liver tissues of 16- and 42-week-old ${ }^{\mathrm{y}} \mathrm{KK}$ mice. D. DNMT1 expression by T2D development duration in mice. $D N M T 1$ expression is not different in 6- and 16-week-old $\mathrm{KK}$ and ${ }^{\mathrm{y}} \mathrm{KK}$ mice. In 42-week-old mice, DNMT1 expression is significantly higher in ${ }^{\mathrm{y} K K}$ than in KK mice $(2.44 \pm 0.7$ fold, $P<0.05)$.

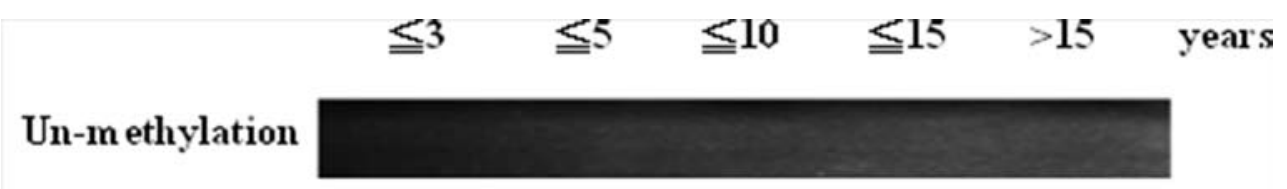

Methylation

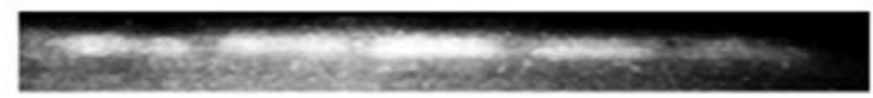

\begin{tabular}{|c|c|c|c|c|c|}
\hline & $\leqq 3$ & $\leqq 5$ & $\leqq 10$ & $\leqq 15$ & $>15$ \\
\hline Un-methylation & - & - & - & - & - \\
\hline Methylation & + & + & + & + & + \\
\hline
\end{tabular}

Figure 4: PTPRD methylation during disease development. The 94 patients with T2D were divided into 5 groups according to the disease duration, (1) less than 3 years $(N=19)$, (2) less than 5 years $(N=16)$, (3) less than 10 years $(N=27),(4)$ less than 15 years $(N=17)$, and (5) longer than 15 years $(N=15)$. PTPRD is methylated in every disease stage in patients.

with susceptibility to T2D (Table 1). Moreover, DNMT1 mRNA expression was higher in patients than in controls (relative expression was 2.23, $P<0.05$, Fig. 5).

\section{DISCUSSION}

GWAS data can reveal markers that may explain clinical and pathological specifics of T2D in a given population. Tsai et al. [9] found that the PTPRD gene was associated with susceptibility to T2D in Han Chinese. T2D risk alleles in PPAR 2 (rs1801282) and PTPRD (rs17584499) are associated with the therapeutic efficacy of pioglitazone $[14,15]$. In this study, we showed that PTPRD mRNA levels were lower in patients with T2D and that they were correlated with disease progression. Until now, no correlation of T2D disease with genetic and environmental factors or with epigenetic regulation has been found.

Cells overexpressing PPAR 2 were used to examine PTPRD function in insulin signaling pathway in T2D. We found that PPAR 2 expression caused PTPRD overexpression in an insulin receptor-sensitive manner. We also found that PTPRD knockdown induced the 
Table 1: Genotypic and allelic frequencies of DNMT1 genetic polymorphism in patients with T2D and controls

\begin{tabular}{|c|c|c|c|c|c|}
\hline \multicolumn{2}{|l|}{ dbSNP ID } & \multirow{2}{*}{$\begin{array}{c}\text { T2D } \\
\text { Total }=1924(\%)\end{array}$} & Control & \multirow[t]{2}{*}{ OR $(95 \%$ CI $)$} & \multirow[t]{2}{*}{$P$-value } \\
\hline rs78789647 & Genotype & & Total $=3602(\%)$ & & \\
\hline & TT & $10(0.52)$ & $10(0.28)$ & $1.92(0.80-4.63)$ & 0.035 \\
\hline & TC & 271(14.09) & $435(12.08)$ & & \\
\hline & $\mathrm{CC}$ & $1643(85.39)$ & $3157(87.64)$ & Ref & \\
\hline & Allele frequency & & & & \\
\hline & $\mathrm{T}$ & 291(7.56) & $455(6.32)$ & $1.21(1.04-1.41)$ & 0.013 \\
\hline & $\mathrm{C}$ & $3557(92.44)$ & 6749(93.68) & Ref & \\
\hline
\end{tabular}

CI, confidence interval; OR, odds ratio.

${ }^{\mathrm{a}} \mathrm{Genotype}$ distribution between patients and control were calculated by $2 \times 3$ chi square test.

down-regulation of the insulin receptor. PPAR 2 gene expression was previously shown to be associated with T2D [16]. PPAR $\gamma 2$ belongs to the nuclear hormone receptor superfamily of ligand-dependent transcription factors [17] and is mainly expressed in adipose tissue [18]. Thiazolidinedione (TZD) is an insulin-sensitizing drug, which is widely used for patients with T2D [19, 20] and works mainly as an agonist of the nuclear receptor PPAR $\gamma$ [21]. The binding of TZD to PPAR $\gamma 2$ activates the transcription of genes involved in glucose and lipid metabolism [22]. These results indicate that PTPRD is involved in the insulin signaling pathway. STAT3, a wellknown oncoprotein [23], was inactivated by $P T P R D$ activation and STAT3 was overexpression while PTPRD was inhibited [11], our results showed that PTPRD was well functional in the pathway.

KK-Ay mice, also known as Yellow KK obese mice, carry the lethal yellow obese $\left(\mathrm{A}^{\mathrm{y}}\right)$ mutation and develop diabetes of polygenic origin, show severe obesity, hypertriglyceridemia, hyperglycemia, hyperinsulinemia, and glucose intolerance by 8 weeks of age $[24,25]$. Therefore, they are widely used as an experimental model for obesity and type 2 diabetes. In the current study, we used early stage mice, before 8 weeks of age, middle stage after 8 weeks of age, and later stage at 42 weeks of age for our experiments. We examined PTPRD expression at different time points during the disease progression. Results indicated that PTPRD mRNA and protein expression were lower in middle stage and later stage T2D mice than in controls. These results were similar to those obtained for patients with T2D. We found PTPRD promoter hypermethylation in middle and late disease stages in mice, but not in early stage and normal mice, similarly to the results found in patients. The PTPRD gene is frequently subject to cancer-specific epigenetic silencing via promoter CpG island DNA hypermethylation [12]. This suggests that PTPRD might be epigenetically silenced by DNA methylation in T2D as well. DNA methylation of $\mathrm{CpG}$ sites is the most common mechanism of epigenetic gene silencing. In cancer, aberrant methylation often results in the inactivation of tumor suppressor genes [26]. Several studies have identified tumor-specific methylation of $\mathrm{CpG}$ islands within the $5^{\prime}$ end of the regulatory region of the genes encoding the receptor-type PTPs such as PTPRD [12] in a wide range of adenocarcinomas, glioblastomas, and squamous cell carcinomas [27]. Our result is the first report to show the correlation between PTPRD and T2D disease development.

DNA methylation in humans occurs predominantly in the context of $\mathrm{CpG}$ dinucleotides [28]. The enzymes responsible for this modification, DNA methyltransferases (DNMTs), are well-characterized and conserved in mammals [28]. DNMTs fall under two categories: de novo and maintenance [29]. Patterns of DNA methylation are initially established by the de novo DNA methyltransferases, DNMT3A and DNMT3B, during the blastocyst stage of embryonic development [30, 31]. Our results showed that $D N M T 3 A, D N M T 3 B$, and $D N M T 3 L$ expression was not different in T2D and control mice. However, DNMT1 expression was significantly higher in T2D mice and patients than in controls. We also found a DNMT1 SNP (rs78789647) that correlated with susceptibility to T2D. DNMT1 shows a preference for hemi-methylated DNA [32-34]. Both the establishment and maintenance of DNA methylation patterns are crucial for development, as DNMT3B- or DNMT1-deficiency in mice results in embryonic lethality [31,35]. De novo DNA methylation in many human primary tumors is initiated within exon 1 of tumor-suppressor genes and subsequently spreads to the promoter region [36]. Using a multifaceted genomic analysis approach, we determined that $P T P R D$ is inactivation via both genetic and epigenetic mechanisms in $\mathrm{T} 2 \mathrm{D}$.

In this study, we found the first evidence that PTPRD levels are significantly lower in patient with $\mathrm{T} 2 \mathrm{D}$, that this protein is involved in the insulin signaling pathway, and 


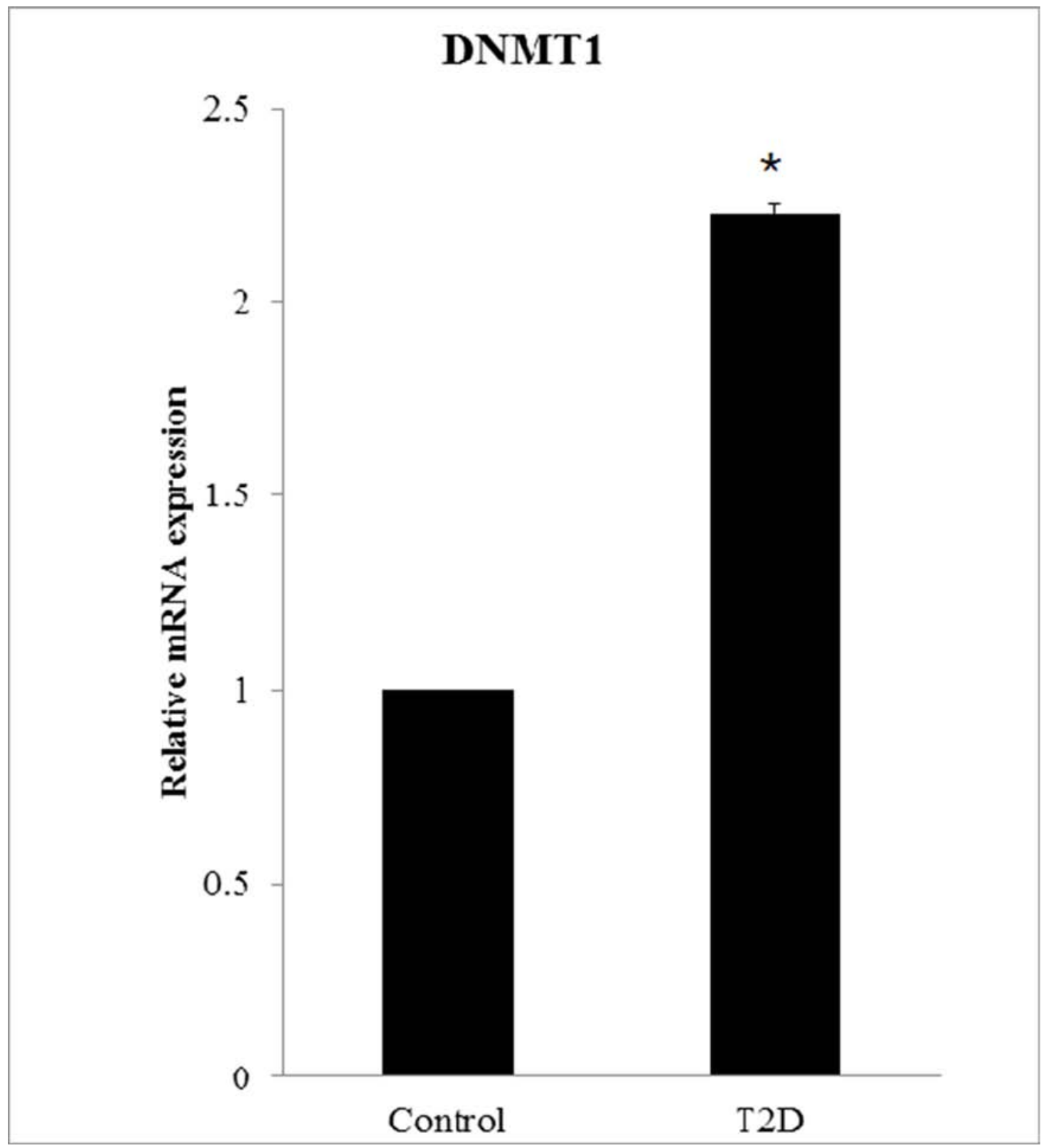

Figure 5: DNMT1 overexpression in patients with T2D. $D N M T 1$ expression is significantly higher in patients with T2D $(N=94)$ than in controls $(N=98)$. The relative mRNA expression level in patients is $2.23 \pm 0.025$.

that it can be epigenetically silenced by DNA methylation. We also found that DNMT1 increases PTPRD DNA methylation during T2D development. These findings increase the understanding of the crucial roles of the regulatory elements in human T2D susceptibility.

\section{MATERIALS AND METHODS}

\section{Patients and sample collection for quantitative real-time PCR (qRT-PCR) analysis}

A total of 192 blood samples (98 controls and 94 patients with T2D) were collected. All individuals attended the China Medical University Hospital in Taichung and fulfilled the diagnostic criteria for T2D. Total RNA was isolated from human blood using the High Pure RNA Isolation Kit (Roche, Mannheim, Germany) according to the manufacturer's instructions. cDNA was synthesized from $1 \mu \mathrm{g}$ total RNA, using the High Capacity cDNA reverse transcription kit (Applied Biosystems, Foster City, CA, USA), in a $20-\mu \mathrm{L}$ reaction volume, according to the manufacturer's instructions. cDNA was diluted to $10 \mathrm{ng} / \mathrm{L}$, and $1 \mu \mathrm{L}$ cDNA was used for each qRT-PCR reaction in a final reaction volume of $10 \mu \mathrm{L}$. For quantification of gene expression with the ABI ViiA $^{\text {TM }} 7$ Real-Time PCR System (Applied Biosystems), 
the FastStart Universal SYBR Green Master mix (Roche) was used. Primer sequences were as follows: PTPRD sense: 5'-TTTACACGAACACCCGTTGA-3', antisense: 5'-CGGAGTCCGTAAGGGTTGTA-3'; and GAPDH sense: 5'-CAGCCTCAAGATCATC AGCA- 3', antisense: 5'-TGTGGTCATGAG TCCTTCCA-3'. This study was approved by the Human Studies Committee of China Medical University Hospital, and informed consent was obtained from either the participants or their parents.

\section{Genomic DNA extraction and MSP}

Genomic DNA was extracted from peripheral blood leukocytes by using the Genomic DNA extraction kit (Qiagen, Valencia, CA, USA), according to the manufacturer's instructions. Bisulfite modification of genomic DNA was carried out using the EZ DNA methylation Kit (Zymo Research, Irvine, CA, USA). Primer sequences used for PTPRD MSP were as follows: 5'-TGTGGGGGTAGTGTTTTGTTTTG-3'; 5'-ACTCTC CCCACCAAAACTAACTAACA-3'; 5'-TGGGGGTAGC GTTTCGTTTC-3'; and 5'-CCCGCCGAAAC TAACTA ACG-3' [10].

\section{Transfections}

HepG2 cells were seeded at a density of 150,000 cells per well on six-well culture plates and incubated until the culture reached $50-80 \%$ confluence. Cells were then transfected with either the empty pSG5 vector, pSG5-hPPAR 2 (provided by Professor Haw-Wen Chen, Department of Nutrition, China Medical University, Taichung, Taiwan), or the lentiviral expression system for PTPRD shRNA (provided by the National RNAi Core Facility, Academia Sinica, Taiwan), by using the Xfect Transfection Reagent (Clontech, Palo Alto, CA, USA), according to the manufacturer's instructions. After $24 \mathrm{~h}$ of transfection, total RNA and protein were isolated from the cells.

\section{Protein extraction and western blots}

The cells were homogenized in ice-cold radioimmunoprecipitation (RIPA) lysis buffer (Millipore, Temecula, CA, USA) with freshly added protease inhibitor and phosphatase inhibitor (FIVEphoton, San Diego, CA, USA). The homogenate was incubated on ice for $30 \mathrm{~min}$ and centrifuged at $13,000 \mathrm{~g}$ for $30 \mathrm{~min}$ at $4{ }^{\circ} \mathrm{C}$. The supernatant was used for western blotting. Proteins $(40 \mu \mathrm{g})$ were separated by SDS-PAGE using a 10\% acrylamide resolving gel and transferred to a polyvinylidene difluoride membrane. Membranes were incubated in a blocking solution containing 5\% nonfat dry milk and $0.1 \%$ Tween-20 in Tris-buffered saline, followed by incubation with a rabbit anti-PTPRD (1:1, 000; LifeSpan BioSciences, Seattle, WA, USA), anti-PPAR (1:1,000; LifeSpan BioSciences), anti-insulin receptor $(1: 1,000$;
GeneTex, Irvine, CA, USA), anti-STAT3 (1:1,000; GeneTex), and anti-actin (1:5, 000; GeneTex) polyclonal primary antibodies. Membranes were then incubated with horseradish peroxidase-conjugated goat anti-rabbit IgG (1:5,000; Jackson ImmunoResearch, West Grove, PA, USA) secondary antibody. Proteins were visualized using SuperSignal West Pico Chemiluminescent Substrate or SuperSignal West Femto Chemiluminescent Substrate (Thermo, Rockford, IL, USA).

\section{Animals}

Four-week-old male $\mathrm{KK}$ and $\mathrm{KK}-\mathrm{Cg}-A^{y} / \mathrm{J}$ mice were obtained from the Jackson Laboratory (Bar Harbor, Maine, USA). Six-, 16-, and 42-week-old mice were used for analyses. Animals were housed in individual cages and fed lab chow ad libitum (LabDiet 5k52, St. Louis, MO, USA). The animals were housed in a room with a conditioned temperature $\left(22-25^{\circ} \mathrm{C}\right)$, relative humidity (50-70\%), and photoperiod (12-h light/12-h dark). This study was approved by the Institutional Animal Care and Use Committee (IACUC) of China Medical University (IACUC: 102-217).

\section{Immunohistochemistry}

Immunohistochemical staining of PTPRD was performed using rabbit anti-PTPRD (LifeSpan BioSciences) and a streptavidin-biotin-peroxidase complex. After deparaffinization and rehydration, formalin-fixed paraffin-embedded tissue sections were incubated in $3 \% \mathrm{H}_{2} \mathrm{O}_{2}$ in distilled water for $30 \mathrm{~min}$ at room temperature, followed by antigen retrieval by boiling the sections in $0.01 \mathrm{M}$ citrate buffer for $20 \mathrm{~min}$. Sections were then washed in $50 \mathrm{mM}$ Tris- $\mathrm{HCl}$ ( $\mathrm{pH} 7.6$ ) with $0.05 \%$ Tween for $2 \mathrm{~min}$. To block nonspecific binding, all sections were incubated with $5 \%$ nonfat dry milk for $30 \mathrm{~min}$ at room temperature. The slides were then incubated with the primary antibody antiPTPRD (1:500) for $1 \mathrm{~h}$ at room temperature. The reaction was stopped by rinsing the sections with $0.01 \mathrm{M}$ PBS. The sections were then incubated with biotinylated antimouse/rabbit IgG serum (secondary antibody), followed by treatment with a peroxidase-labeled streptavidin-biotin complex and diaminobenzidine substrate to visualize the positive cells. Finally, sections were counterstained with hematoxylin, prior to being mounted for examination by light microscopy. PTPRD-positive cells per area in the mouse liver were counted using the Q500MC Image Analysis System (Leica, Nussloch, Germany). PTPRDpositive cells were quantified in 20 fields from the cortex and 15 from the medulla at a magnification of $200 \times$.

\section{Patients and sample collection for genotyping}

We enrolled 1938 patients in this study. All individuals attended the China Medical University 
Hospital in Taichung and fulfilled the diagnostic criteria for T2D. To compare the prevalence of polymorphisms in patients with that in a healthy population, we downloaded the genotype frequency data of 3,602 healthy controls from the Taiwan Biobank (https://taiwanview.twbiobank .org.tw/taiwanview/dl.do). The SNPs in the target gene were queried from the NCBI dbSNP database (http://www.ncbi.nlm.nih.gov/snp). We obtained the SNPs of our gene of interest and compared the SNPs in the diseased and control group. Chi-square tests were used to calculate odds ratios and $P$ values. The study was approved by the institutional review board of the China Medical University Hospital.

\section{Statistical analysis}

Data were assembled using the Microsoft Excel software and analyzed using the SPSS 15.0 (SPSS, Chicago, IL, USA) or the GraphPad Prism version 3 (GraphPad Software, San Diego, CA, USA) statistical packages. All values were expressed as means ( \pm standard deviation (SD)). Normality of the data was tested using the Kolmogorov-Smirnov test. Hierarchical gene analysis and heat maps were determined using a Pearson correlation matrix. Depending on the probability distribution pattern and the total number of subjects, parametric (normal distribution and $\geq 50$ subjects) or non-parametric tests (skewed distribution or $<50$ subjects) were used. Levels of significance were set at $P<0.05$ (two-tailed).

\section{FUNDING SOURCES}

This work was supported in part by China Medical University Hospital (DMR-99-050, DMR-100-060, DMR103-039, and DMR-104-102) in Taiwan. The first author received a fellowship from China Medical University (CMU 101-AWARD-01), Taiwan.

\section{CONFLICTS OF INTEREST}

The authors declare no competing interest.

\section{REFERENCES}

1. Stumvoll M, Goldstein BJ, van Haeften TW. Type 2 diabetes: principles of pathogenesis and therapy. Lancet. 2005; 365:1333-1346.

2. Liao WL, Tsai FJ. Personalized medicine in Type 2 Diabetes. BioMedicine. 2014; 4:1-8.

3. Gunasekaran U, Gannon M. Type 2 diabetes and the aging pancreatic beta cell. Aging (Albany NY). 2011; 3:565-575.

4. Ozanne SE, Sandovivi I, Constancia M. Maternal diet, aging and diabetes meet at a chromatin loop. Aging (Albany NY). 2011; 3:548-554.
5. Ledermann HM. Maturity-onset diabetes of the young (MODY) at least ten times more common in Europe than previously assumed? Diabetologia. 1995; 38:1482.

6. Maassen JA, T Hart LM, Van Essen E, Heine RJ, Nijpels G, Jahangir Tafrechi RS, Raap AK, Janssen GM, Lemkes HH. Mitochondrial diabetes: molecular mechanisms and clinical presentation. Diabetes. 2004; 53:S103-109.

7. Fajans SS, Bell GI, Polonsky KS. Molecular mechanisms and clinical pathophysiology of maturity-onset diabetes of the young. N Engl J Med. 2001; 345:971-980.

8. Moore AF, Florez JC. Genetic susceptibility to type 2 diabetes and implications for antidiabetic therapy. Annu Rev Med. 2008; 59:95-111.

9. Tsai FJ, Yang CF, Chen CC, Chuang LM, Lu CH, Chang CT, Wang TY, Chen RH, Shiu CF, Liu YM, Chang $\mathrm{CC}$, Chen $\mathrm{P}$, Chen $\mathrm{CH}$, et al. A genome-wide association study identifies susceptibility variants for type 2 diabetes in Han Chinese. PLoS Genet. 2010; 6:e1000847.

10. Hunter T. Signaling -2000 and beyond. Cell. 2000; 100:113-127.

11. Ortiz B, Fabius AW, Wu WH, Pedraza A, Brennan CW, Schultz N, Pitter KL, Bromberg JF, Huse JT, Holland EC, Chan TA. Loss of the tyrosine phosphatase PTPRD leads to aberrant STAT3 activation and promotes gliomagenesis. Proc Natl Acad Sci U S A. 2014; 111:8149-8154.

12. Veeriah S, Brennan $C$, Meng S, Singh B, Fagin JA, Solit DB, Paty PB, Rohle D, Vivanco I, Chmielecki J, Pao W, Ladanyi M, Gerald WL, et al. The tyrosine phosphatase PTPRD is a tumor suppressor that is frequently inactivated and mutated in glioblastoma and other human cancers. Proc Natl Acad Sci U S A. 2009; 106:9435-9440.

13. Chang YC, Chiu YF, Liu PH, Shih KC, Lin MW, Sheu WH, Quertermous T, Curb JD, Hsiung CA, Lee WJ, Lee PC, Chen YT, Chuang LM. Replication of genome-wide association signals of type 2 diabetes in Han Chinese in a prospective cohort. Clin Endocrinol. 2012; 76:365-372.

14. Pei Q, Huang Q, Yang GP, Zhao YC, Yin JY, Song M, Zheng Y, Mo ZH, Zhou HH, Liu ZQ. PPAR-gamma2 and PTPRD gene polymorphisms influence type 2 diabetes patients' response to pioglitazone in China. Acta pharmacol Sin. 2013; 34:255-261.

15. Maruthur NM, Gribble MO, Bennett WL, Bolen S, Wilson LM, Balakrishnan P, Sahu A, Bass E, Kao WH, Clark JM. The pharmacogenetics of type 2 diabetes: a systematic review. Diabetes Care. 2014; 37:876-886.

16. Altshuler D, Hirschhorn JN, Klannemark M, Lindgren CM, Vohl MC, Nemesh J, Lane CR, Schaffner SF, Bolk S, Brewer C, Tuomi T, Gaudet D, Hudson TJ, et al. The common PPARgamma Pro12Ala polymorphism is associated with decreased risk of type 2 diabetes. Nat Genet. 2000; 26:76-80.

17. Issemann I, Green S. Activation of a member of the steroid hormone receptor superfamily by peroxisome proliferators. Nature. 1990; 347:645-650. 
18. Auwerx J. PPARgamma, the ultimate thrifty gene. Diabetologia. 1999; 42:1033-1049.

19. Willson TM, Lambert MH, Kliewer SA. Peroxisome proliferator-activated receptor gamma and metabolic disease. Annu Rev Biochem. 2001; 70:341-367.

20. Zimmet P. Addressing the insulin resistance syndrome: a role for the thiazolidinediones. Trends Cardiovasc Med. 2002; 12:354-362.

21. Soccio RE, Chen ER, Lazar MA. Thiazolidinediones and the Promise of Insulin Sensitization in Type 2 Diabetes. Cell Metab. 2014; 20:573-591.

22. Miyazaki Y, Mahankali A, Matsuda M, Mahankali S, Hardies J, Cusi K, Mandarino LJ, DeFronzo RA. Effect of pioglitazone on abdominal fat distribution and insulin sensitivity in type 2 diabetic patients. J Clin Endocrinol Metab. 2002; 87:2784-2791.

23. Bromberg JF, Wrzeszczynska MH, Devgan G, Zhao Y, Pestell RG, Albanese C, Darnell JE Jr. Stat3 as an oncogene. Cell. 1999; 98:295-303.

24. Iwatsuka H, Shino A, Suzuoki Z. General survey of diabetic features of yellow KK mice. Endocrinol Jpn. 1970; $17: 23-35$.

25. Castle CK, Colca JR, Melchior GW. Lipoprotein profile characterization of the $\mathrm{KKA}(\mathrm{y})$ mouse, a rodent model of type II diabetes, before and after treatment with the insulinsensitizing agent pioglitazone. Arterioscler Thromb. 1993; 13:302-309.

26. Esteller M. Epigenetics in cancer. N Engl J Med. 2008; 358:1148-1159.

27. Jacob ST, Motiwala T. Epigenetic regulation of protein tyrosine phosphatases: potential molecular targets for cancer therapy. Cancer Gene Ther. 2005; 12:665-672.
28. Law JA, Jacobsen SE. Establishing, maintaining and modifying DNA methylation patterns in plants and animals. Nat Rev Genet. 2010; 11:204-220.

29. Goll MG, Bestor TH. Eukaryotic cytosine methyltransferases. Annu Rev Biochem. 2005; 74:481-514.

30. Okano M, Xie S, Li E. Cloning and characterization of a family of novel mammalian DNA (cytosine-5) methyltransferases. Nat Genet. 1998; 19:219-220.

31. Okano M, Bell DW, Haber DA, Li E. DNA methyltransferases Dnmt3a and Dnmt3b are essential for de novo methylation and mammalian development. Cell. 1999; 99:247-257.

32. Bestor TH, Ingram VM. Two DNA methyltransferases from murine erythroleukemia cells: purification, sequence specificity, and mode of interaction with DNA. Proc Natl Acad Sci U S A. 1983; 80:5559-5563.

33. Bestor T, Laudano A, Mattaliano R, Ingram V. Cloning and sequencing of a cDNA encoding DNA methyltransferase of mouse cells. The carboxyl-terminal domain of the mammalian enzymes is related to bacterial restriction methyltransferases. J Mol Biol. 1988; 203:971-983.

34. Hermann A, Goyal R, Jeltsch A. The Dnmt1 DNA(cytosine-C5)-methyltransferase methylates DNA processively with high preference for hemimethylated target sites. J Biol Chem. 2004; 279:48350-48359.

35. Li E, Bestor TH, Jaenisch R. Targeted mutation of the DNA methyltransferase gene results in embryonic lethality. Cell. 1992; 69:915-926.

36. Nguyen C, Liang G, Nguyen TT, Tsao-Wei D, Groshen S, Lubbert M, Zhou JH, Benedict WF, Jones PA. Susceptibility of nonpromoter $\mathrm{CpG}$ islands to de novo methylation in normal and neoplastic cells. J Natl Cancer Inst. 2001; 93:1465-1472. 